\title{
MBE Growth and Characterization of a III-V Distributed Bragg Reflectors and InAs Quantum Dots
}

\author{
J.-G. Rousset, T. SŁupinski, T. Jakubczyk, J. Kobak, P. Stawicki, K. GoŁasa, \\ A. Babinski, M. Nawrocki and W. PaCuski \\ Institute of Experimental Physics, Faculty of Physics, University of Warsaw \\ Hoża 69, PL-00-681 Warszawa, Poland
}

\begin{abstract}
We describe the realization and characterization of a distributed Bragg reflectors and InAs quantum dots grown by molecular beam epitaxy. The distributed Bragg reflectors are based on a stack of eight or twenty pairs of GaAs and AlAs layers with a stopband centered at about $E_{0}=1.24 \mathrm{eV}\left(\lambda_{0}=1000 \mathrm{~nm}\right)$. The whole structures exhibit a reflectivity coefficient above $90 \%$. The growth rate was monitored in situ by measurement of the oscillations of the thermal emission intensity. The investigations conducted on the InAs quantum dots grown on GaAs show photoluminescence around $E=1.25 \mathrm{eV}(\lambda=990 \mathrm{~nm})$. The combination of these two elements results in the realization of a microcavity containing InAs quantum dots and surrounded by 20 pairs of distributed Bragg reflectors.
\end{abstract}

PACS: 78.20.Ci, 78.67.Pt, 68.37.Hk

\section{Introduction}

A distributed Bragg reflector (DBR) is a one-dimensional photonic structure consisting of a stack of alternating high and low refractive index layers. In such a structure, the boundary conditions for each layer interface causes a partial reflection of the electromagnetic waves and the interferences resulting from the multiple reflections from all the layers are constructive [1]. Thus, one can obtain a high quality reflector for a range of wavelengths defining the stopband of the structure. The key parameter for obtaining high quality monolithic structures is the lattice matching of the different layers to avoid the effects of strain such as misfit dislocations [2-4].

A DBR can be made of any transparent material, however, using semiconductors allows one to utilize DBRs as an element for optic and optoelectronic devices. The realization of high quality DBRs is of large interest for the achievement of a wide range of optoelectronic devices such as vertical cavity surface emitting lasers (VCSELs) [5] or resonant cavity light emitting diodes (RCLEDs) [6]. The main advantages of VCSELs compared to classical edge emitting lasers is the possibility to process tens of thousands of VCSELs simultaneously on a three inches wafer; in addition, the device quality can be tested at several stages of the production process. Furthermore, the high reflectivity DBRs reduce the threshold current resulting in a lower power consumption [7]. These features make VCSELs of particular interest for optical fiber data communication.

In this work, we present the realization of the first structures grown by MBE at the University of Warsaw. The growth of the DBRs and microcavity are monitored by measurements of the oscillation of the sample's thermal emission from which we deduce the reflectivity, and consequently the layer thickness and the growth rate.

\section{Growth and characterization}

The growth by MBE method and initial characterization of the DBR and the InAs quantum dots (QDs) were the starting point for the design of an integrated structure consisting of an optically active microcavity with InAs quantum dots surrounded by high quality DBRs presented in Sect. 2.3 of this article. The two growth chambers (one for II-VI and one for III-V compounds) MBE machine used to grow our structures was delivered by SVT Associates.

\subsection{8 pairs $D B R$}

The DBR was grown on a GaAs substrate (100) on which was deposited a $500 \mathrm{~nm}$ GaAs buffer followed by the $\lambda /(4 n)$ AlAs and GaAs layers constituting the 8-fold DBR. The observation of the RHEED reconstruction pattern ensured us that the grown layers are of good crystalline quality. The structure was designed so that the stopband is centered at about $E_{0}=1.31 \mathrm{eV}$ $\left(\lambda_{0}=950 \mathrm{~nm}\right)$. Knowing the values of the refractive index at this wavelength for GaAs $(n=3.54)$ and AlAs $(n=2.96)$ we determined the thicknesses of the layers $(d=\lambda / 4 n): d_{\mathrm{GaAs}}=67.1 \mathrm{~nm}$ and $d_{\mathrm{AlAs}}=80.2 \mathrm{~nm}$. The substrate during growth was monitored by thermal emission measurements at $\lambda=950 \mathrm{~nm}$. Such measurements are typically used for the determination of the substrate temperature, but it is disturbed by the interferences in 
thin epitaxial layers. In our study, we used the effect of the interferences for the determination of the layer thickness and the growth rate. The period of the oscillations of the thermal radiation corresponds to the growth of a $\lambda /(4 n)$ thick layer.

After growth the DBR was observed under scanning electron microscope (SEM). As seen in Fig. 1 the layers have regular thickness and the interfaces are smooth which attests to the good growth conditions. Reflectivity measurements conducted at room temperature (see Fig. 2) show that the stopband is centered at $E_{0}=$ $1227 \mathrm{meV}\left(\lambda_{0}=1010 \mathrm{~nm}\right)$ with a maximum reflectivity of $92 \%$. The measured width of the stopband is $150 \pm 30 \mathrm{meV}(130 \pm 25 \mathrm{~nm})$, in coherence with the theoretical value given $[1,8]$ by Eq. (1) with a refractive index step $\Delta n=0.57$ and mean refractive index $\tilde{n}=3.23$ which gives a theoretical stopband $\Delta \lambda_{\mathrm{th}}=112 \mathrm{~nm}$.

$$
\frac{\Delta \lambda_{\mathrm{th}}}{\lambda_{0}}=\frac{4}{\pi} \sin ^{-1}\left(\frac{\left|n_{2}-n_{1}\right|}{n_{1}+n_{2}}\right) \approx \frac{2}{\pi} \frac{\Delta n}{\tilde{n}} .
$$

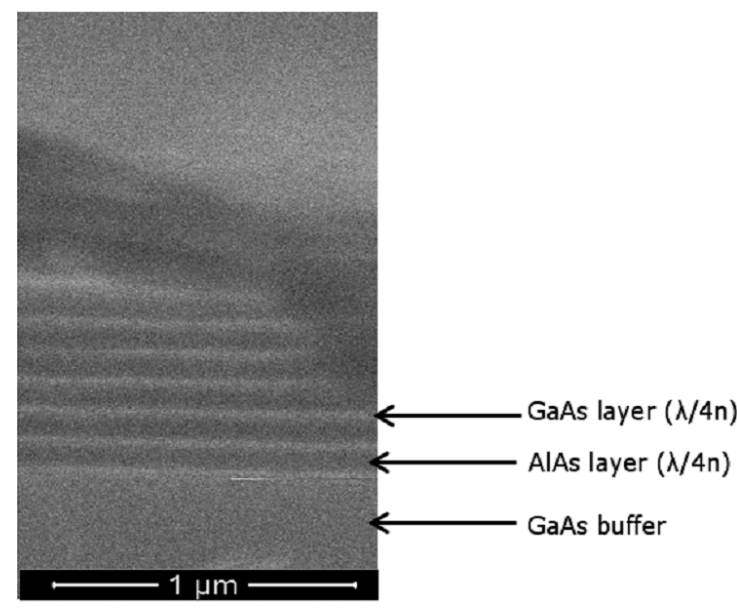

Fig. 1. Scanning electron microscopy imaging of the 8 pairs DBR. Picture taken at the edge of the sample.

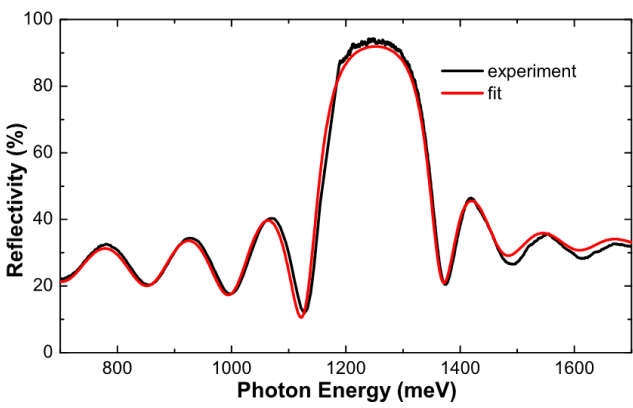

Fig. 2. Measured and fitted reflectivity of the 8 pairs DBR. The fit was realized using the transfer matrix method. The maximum reflectivity is $92 \%$ and the stopband is $150 \mathrm{meV}$ (130 nm) wide.

For a 1D photonic structure with steep changes of the refractive index, $n_{i}$ is the refractive index of the layer $i$, $\tilde{n}$ - the average refractive index, $\Delta \lambda$ - the width of the stopband and $\lambda_{0}$ - the center of the stopband.

\subsection{InAs/GaAs quantum dots}

Self-assembled InAs QDs were grown by MBE on GaAs substrate. After growth of a $250 \mathrm{~nm}$ GaAs buffer and the control of the crystal quality by observation of the RHEED pattern, two techniques were used to grow InAs QDs. Droplet epitaxy consists of depositing a thin layer of metal ( $\approx 2 \mathrm{ML}$ of In in our case) that forms droplets at the surface of the grown structure. In a next step, the exposition under As flux induces the crystallization and formation of the quantum dots [9]. The second method consists of direct deposition of InAs on the GaAs buffer $[10,11]$. The parameters were chosen so that $\approx 1.8 \mathrm{ML}$ of InAs is deposited. A $40 \mathrm{~nm}$ GaAs cap was then grown over the QDs.

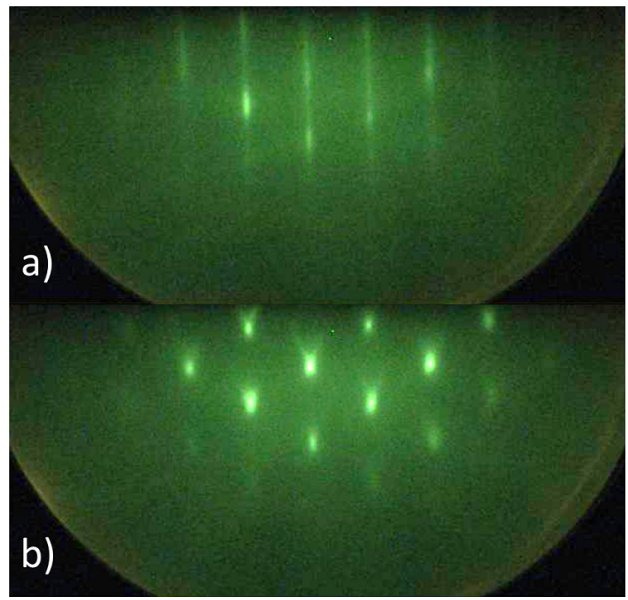

Fig. 3. RHEED imaging obtained during the growth of InAs/GaAs QDs: (a) 2D pattern for less than 1.6 monolayer deposited, (b) 3D pattern for more than 1.6 monolayer deposited. The amount of InAs is $\approx 2 \mathrm{ML}$ for the droplet epitaxy method and $\approx 1.8 \mathrm{ML}$ for direct deposition of InAs.

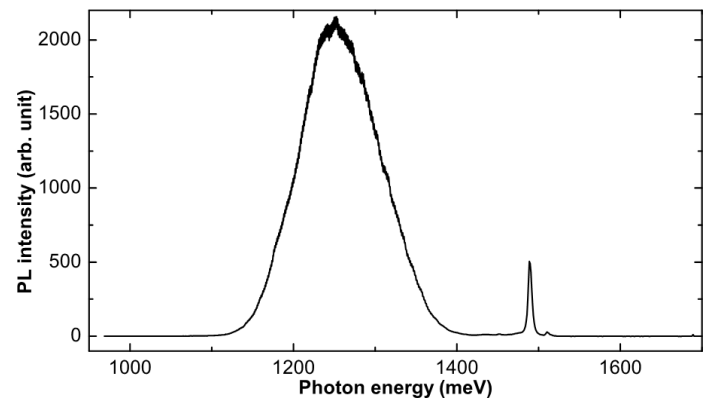

Fig. 4. Photoluminescence spectrum of the InAs/ GaAs QDs exhibiting emission centered at $E_{0}=$ $1250 \mathrm{meV}$ in the same range as the stopband of the DBR.

The formation of the QDs was monitored by the transition of the RHEED pattern from $2 \mathrm{D}$ to $3 \mathrm{D}$ (see Fig. 3 ) 
after the deposition of 1.6 ML of InAs. The grown QDs have been characterized by photoluminescence measurements conducted at liquid helium temperature in a continuous flow helium cryostat. The spectra registered show intense emission centered at $E_{0}=1250 \mathrm{meV}$ (see Fig. 4) around the center of the stopband of the DBR previously presented.

\subsection{Microcavity with InAs QDs}

We used the two block elements described previously in the realization of an optically active microcavity consisting of InAs QDs embedded between two 20 fold DBRs.

The structure of the microcavity shown in Fig. 5 was designed with 20-fold DBRs to enhance the light confinement and coupling with the InAs QDs in the cavity. The InAs quantum dots are grown in the middle of the $\lambda / n$ wide cavity, where the amplitude of the stationary wave of the electric field is at its maximum.

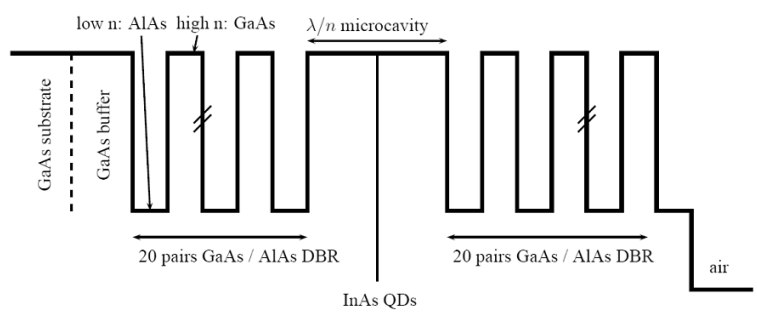

Fig. 5. Structure of the microcavity. Vertical axis: refractive index, horizontal axis: depth of the sample.

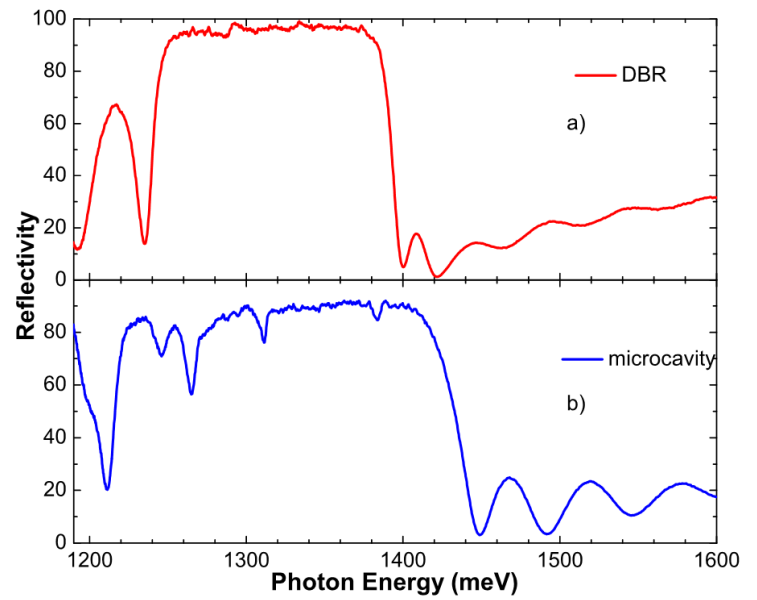

Fig. 6. (a) Reflectivity spectra of the 20 fold GaAs/AlAs DBR centered on $E_{0}=1314 \mathrm{meV}\left(\lambda_{0}=\right.$ $943 \mathrm{~nm}$ ) and (b) of the microcavity containing InAs QDs and surrounded by two 20-fold DBR GaAs/AlAs.

The 20 -fold GaAs/AlAs DBR exhibit a maximum reflectivity of $96 \%$. One can also observe in Fig. 6 a that the stopband maximum is more flat than in the case of 8-pairs DBR, the width of the stopband is similar in both cases. This proves the high quality of the DBR and the

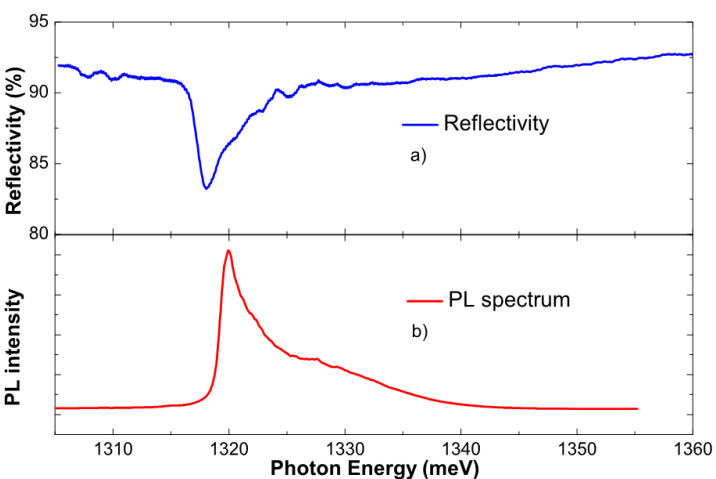

Fig. 7. (a) Close-up on the reflectivity spectrum of the microcavity. (b) Photoluminescence spectrum of the microcavity showing the emission of the cavity mode. The quality factor of the cavity mode is $Q \geqslant 500$.

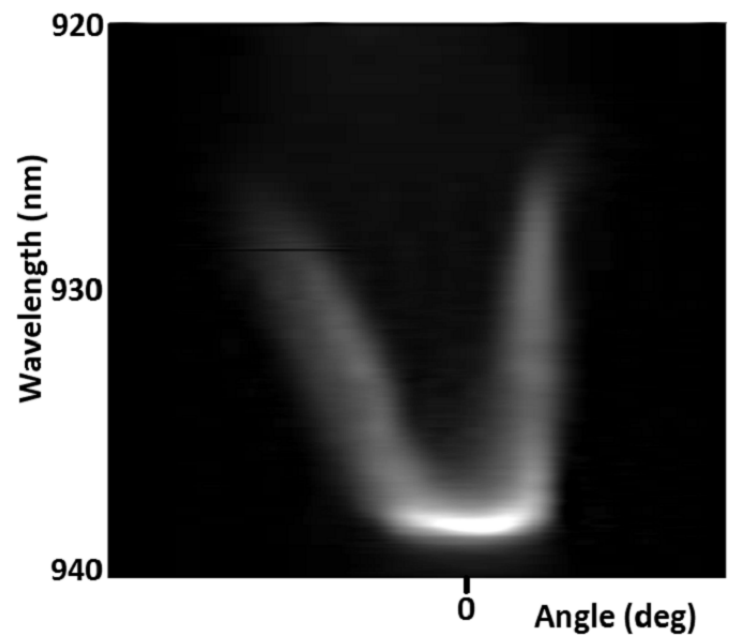

Fig. 8. Angular dispersion of the cavity mode showing the strong dependence of the energy position of the cavity mode on the incidence of the light. The maximum of intensity corresponds to normal incidence of the light.

better reflectivity resulting from the increase of the pair number in the DBR [1]. In Fig. 6b one can see the apparition of the multiple modes of the cavity within the stopband, allowing the coupling of the light with the quantum $\operatorname{dots}[12]$.

Complementary photoluminescence measurements show the light emission of the cavity mode (see Fig. 7 ). This mode has a quality factor [12] $Q=\lambda / \Delta \lambda \geqslant 500$ (in our case).

The far field emission of the cavity mode was collected using the method of the Fourier plane imaging [13] (see Fig. 8). A strong dependence of the energy of the emitted light from the cavity on the emission angle is observed due to the different optical path for the different angles of propagation. It shows that, in the microcavity, the exciton dispersion is much weaker than the photon dispersion. A better quality factor of the cavity mode, 
should result in a stronger coupling of the exciton in the microcavity to the light.

\section{Conclusion}

An eightfold DBR based on GaAs and AlAs layers with a stopband centered at $\lambda_{0}=1000 \mathrm{~nm}$ has been grown by molecular beam epitaxy and the reflectivity spectrum has been explained by a simple model. The monitoring of the oscillations of the thermal emission from the sample during growth, allowed us to efficiently control the thickness of the layers. Two MBE growth methods of InAs/GaAs QDs have been used and the QDs exhibit photoluminescence in the range of the stopband of the DBR previously grown. Resulting from the association of these block elements, an optically active microcavity containing InAs QDs and surrounded by GaAs/AlAs DBRs has been realized. The cavity mode is matched to the emission of QDs and exhibit a quality factor $Q \geqslant 500$.

\section{Acknowledgments}

This work was supported by Polish public funds in years 2011-2014 (NCBiR project LIDER/30/13/L-2/10/ NCBiR/2011). Research was carried out with the use of CePT, CeZaMat and NLTK infrastructures financed by the European Union - the European Regional Development Fund within the Operational Programme "Innovative economy" for 2007-2013.

\section{References}

[1] P. Yeh, Optical Waves in Layered Media, Wiley, New York 1988
[2] W. Pacuski, C. Kruse, S. Figge, D. Hommel, Appl. Phys. Lett. 94, 191108 (2009).

[3] H. Gebretsadik, K. Kamath, K.K. Linder, X. Zhang, P. Bhattacharya, Appl. Phys. Lett. 71, 581 (1997).

[4] F. Genty, G. Almuneau, N. Bertru, L. Chusseau, P. Grech, D. Cot, J. Jacquet, J. Cryst. Growth 183, 15 (1998).

[5] M.E. Murtagh, V. Guenebaut, S. Ward, D. Nee, P.V. Kelly, B. O'Looney, F. Murphy, M. Modreanu, S. Westwater, R. Blunt, S.W. Bland, Thin Solid Films 450, 148 (2004).

[6] V. Vilokkinen, P. Sipila, P. Melanen, M. Saarinen, S. Orsila, M. Dumitrescu, P. Savolainen, M. Toivonen, M. Pessa, Mater. Sci. Eng. B 74, 165 (2000).

[7] K. Iga, IEEE J. Select. Top. Quant. Electron. 6, 1201 (2000).

[8] C.B. Fu, C.S. Yang, M.C. Kuo, Y.J. Lai, J. Lee, J.L. Shen, W.C. Chou, S. Jeng, Chin. J. Phys. 41 535 (2003)

[9] S.F. Tang, S.Y. Lin, S.C. Lee, D. Hommel, J. Nanoparticle Res. 3, 489 (2001).

[10] T. Passow, P. Feinäugle, T. Vallaitis, J. Leuthold, D. Litvinov, D. Gerthsen, M. Hetterich, J. Appl. Phys. 102, 073511 (2007).

[11] P.B. Joyce, T.J. Krzyzewski, T.R. Bell, T.S. Jones, Phys. Rev. B 62, 10891 (2000).

[12] A. Kavokin, J.J. Baumberg, G. Malpuech, F.P. Laussy, Microcavities, Oxford University Press, New York 2011

[13] T. Jakubczyk, W. Pacuski, P. Duch, P. Godlewski, A. Golnik, C. Kruse, D. Hommel, J.A. Gaj, Cent. Eur. J. Phys. 9, 428 (2011). 